\title{
Aims and scope of a new journal on gastric diseases and surgical practice
}

\author{
Vito $D^{\prime}$ Andrea ${ }^{1}$
}

Department of Surgical Sciences, "La Sapienza” University of Rome, Rome 00161, Italy

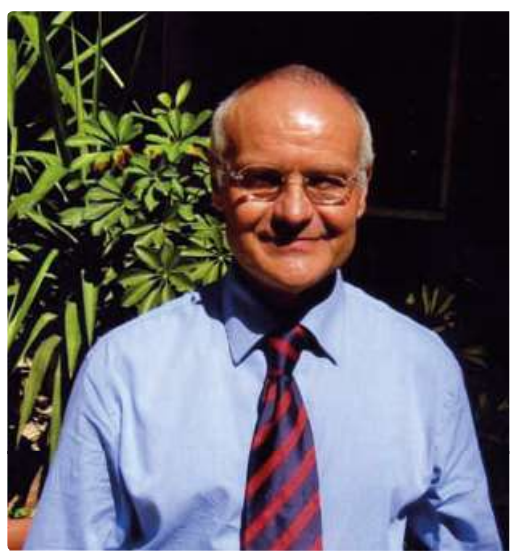

Dear Authors,

the Journal of Gastric Surgery (J Gastr Surg, JGS) is a peer-reviewed journal published and managed by the International Gastric Cancer Unit established between the Department of Surgical Sciences (Sapienza University), Department of Gastric Surgery (Fujian Medical University), Department of Digestive Surgery (St. Mary's Hospital of Terni).

J Gastr Surg publishes original articles, reviews, editorials and letters to the Editor.

Gastric Surgeons all over the world are kindly invited to contribute to the growth of the Journal of Gastric Surgery.

When I was a child, my mother died from inoperable gastric cancer and I decided to dedicate my entire life to surgical research, as I consider that one of the most relevant field in health sciences.

I am the Director of the Department of Surgical Sciences and of the Ph.D. Programme in "Advanced Surgical Technologies" at Sapienza University of Rome.

I decided to found the Journal of Gastric Surgery, together with my Collegues Prof.Amilcare Parisi and Prof.Chang-Ming Huang, in order to publish the advancements in gastric surgery worldwide.

Gastric Cancer is the fourth most common cancer type and the second leading cause of cancer mortality in the world.

Most patients with gastric cancer have advanced stage (III or IV) disease at the time of diagnosis.

Gastrectomy with lymph node dissection still remains the only curative treatment for patients with gastric cancer.

Laparoscopic, open and robotic surgical studies are welcome for publication in the journal.

Gastric Surgery is popular as well in obese patients, since weight loss, diabetes remission, improvement in cardiovascular risk factors and other comorbidities are better achieved with bariatric surgery than medical therapy.

Laparoscopic sleeve gastrectomy and roux-en-y gastric by-pass are the most common procedures.

The metabolic diseases that are most affected by gastric surgery are type 2 diabetes mellitus and the metabolic syndrome. Hypertension and cardiovascular diseases are also improved by gastric surgery through weight loss effects.

The metabolic syndrome is represented by obesity, glucose intolerance, dyslipidemia and hypertension. The metabolic manifestations of the metabolic syndrome respond to surgical therapy.

I'm glad to read your welcome manuscripts,

Yours Sincerely,

\section{Prof. Vito D'Andrea}

UE Cancer Mission Board Member

Full Professor of General Surgery

Director of the Department of Surgical Sciences

"Advanced Surgical Technologies" Ph.D. Director

Sapienza University of Rome, Italy 